\title{
¿Cómo se gobierna el subsuelo político? Gobernabilidades Comunales campesinas en el Catatumbo colombiano
}

Como o subsolo político é governado? Governabilidades comunais camponesas no Catatumbo colombiano

How is the political subsoil governed? Communal peasant governabilites in the colombian Catatumbo

\author{
Andrés LOZANO REYES \\ Universidad Nacional de Colombia (Colombia) \\ anlozanore@unal.edu.co
}

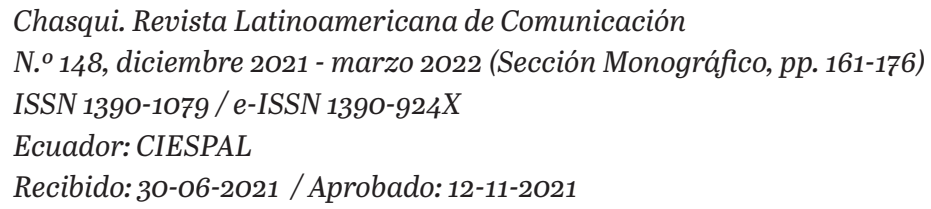




\title{
Resumen
}

El artículo desea contribuir al análisis de las formas de gobierno que se despliegan en el subsuelo político latinoamericano y que están dirigidas según la lógica de la (re)producción de lo común, a través de la categoría de Habilidades Comunales de Gobierno (HCG). A la luz del caso de la Asociación Campesina del Catatumbo, se describen y explican los mecanismos, lógicas y horizontes de comando político campesino, para dar cuenta de las dinámicas del subsuelo político en condiciones de abigarramiento.

Palabras clave: Habilidades Comunales de Gobierno, forma comunitaria de la política, forma comunitaria del trabajo, reproducción de lo común.

\section{Resumo}

$\mathrm{O}$ artigo visa aportar à análise das formas de governo que desenvolvem-se no subsolo político latino-americano e que estão dirigidas de acordo á lógica da (re)produção do comum, por meio da categoria de Habilidades Comunais do Governo (HCG). Á luz do caso da Associação Camponesa do Catatumbo, descrevem-se e explicam-se os mecanismos, lógicas e horizontes de comando politico campones, para dar conta das dinâmicas do subsolo político em condições variegadas.

Palavras-chave: Habilidades Comunais do Governo, forma comunitária da política, forma comunitária do trabalho, reprodução do comum.

\begin{abstract}
The article aims to contribute to the analysis of the forms of government that are deployed in the Latin American political subsoil and that are directed according to the logic of the (re)production of the common, through the category of Communal Skills of Government (CSG). In the light of the case of the Catatumbo Peasant Association, the mechanisms, logics and horizons of peasant political command are described and explained, to account for the dynamics of the political subsoil in conditions of variegation.
\end{abstract}

Keywords: Communal Skills of Government, Communal form of politics, Communal form of labor, (re)production of the common. 


\section{Introducción}

América Latina asiste actualmente a una reactivación de la movilización social y popular, con altos niveles de conflictividad y cuestionamiento al orden social vigente. Este proceso se ha desarrollado con particular intensidad y capacidad innovadora en países que no han transitado por gobiernos progresistas y que se caracterizan por la profundidad y violencia con la que se ha posibilitado la reproducción del modelo neoliberal, como Chile y Colombia (Ouviña, 2020).

En este escenario abierto de disputas por la definición del horizonte social (Estrada et al., 2020) se han desplegado diversos dispositivos y prácticas de resistencia, que van desde ejercicios espontáneos de organización social, hasta la (re)emergencia y reconstitución de sujetos sociales. Estos nuevos movimientos del subsuelo político (Tapia, 2008) no solo resaltan la vitalidad y potencialidad contrahegemónica de los procesos de emancipación regionales, sino que aluden a la necesidad de actualizar y producir pensamiento crítico, capaz de impulsar alternativas societales.

Con este contexto, este artículo presenta la noción de Habilidades Comunales de Gobierno (HCG), como categoría analítica para pensar la expresión concreta de los ejercicios de gobierno para la (re)producción de lo común que se contienen en el subsuelo político y que confrontan, cancelan o cuestionan la lógica de la valorización del valor. En ese sentido, desde el análisis de las HCG desplegadas por la Asociación Campesina del Catatumbo, ${ }^{1}$ se pretende contribuir a la comprensión y producción de conocimiento sobre las dinámicas sociales y políticas regionales, a la vez que se propone una categoría de análisis desde una perspectiva local pero que se plantea en un horizonte latinoamericano de síntesis (Tapia, 2013).

\section{El subsuelo político en América Latina}

Una mirada crítica al proceso de constitución de las sociedades modernas resalta su aparente organicidad. La lógica violenta de desorganización y refuncionalización de otras formas de totalización social, sustentada en la dinámica de separación-concentración de ámbitos de la vida, ha producido un "conjunto de procesos y prácticas en condiciones de marginación y desarticulación” que obstaculizan e interrumpen en diferentes grados la constitución novedosa y realmente orgánica de dichas sociedades (Tapia, 2008, p. 85). Este conjunto de prácticas marginalizadas y negadas sistemáticamente,

1 Este artículo contiene los resultados de la investigación posgradual desarrollada por el autor durante el periodo 2017-2020 para optar al título de Magíster en Estudios Políticos Latinoamericanos de la Universidad Nacional de Colombia y que se desarrolló en el marco del proyecto "Gobernabilidades comunales. Formas de gobierno, gestión y organización del territorio para la construcción de escenarios de paz en Colombia" del Grupo Interdisciplinario de Estudios Políticos y Sociales THESEUS de misma universidad y Ascamcat, 2016-2019. El autor quiere agradecer los comentarios de la profesora Andrea Ávila Serrano (UNAL) en la redacción de este texto 
o subsuelo político, comprende tanto formas comunitarias de la vida como expresiones sociales más contemporáneas y se organiza en tanto "crítica, alternativa, ironía, negación de la institucionalidad política del orden social" (Tapia, 2008, p. 85).

En oposición a la superficie política, o aquel espacio de visibilidad de lo aceptado, lo incluido y lo reconocido por las instituciones hegemónicas, el subsuelo político está constituido por las "prácticas y discursos políticos que no son reconocidos social y estatalmente, pero emergen como forma de asociación, interacción y opinión sobre la dimensión política y de gobierno de las sociedades" y que se reproducen bajo sus propias formas de solidaridad y comunicación (Tapia, 2008, p. 96).

Estos procesos de desconocimiento de pluralidades sociales se desarrollan con particular intensidad en formaciones sociales abigarradas como las latinoamericanas (Zavaleta, 2021), donde coexisten y se sobreponen desarticuladamente "varios tiempos históricos, modos de producción, concepciones del mundo, lenguas, culturas y diferentes estructuras de autoridad" (Tapia, 2010, p. 100). Aunque en la región se hayan alcanzado grados importantes de totalización del modo de producción capitalista o de subsunción real de las formas de vida a la lógica del capital, existen vastas diversidades sociales que no están subsumidas más que formalmente. Estos procesos alternativos de reproducción de la vida han logrado contener la extensión total de las relaciones capitalistas, conservando grados de decisión sobre la dirección de lo social (Tapia, 2017).

Dicha sobreposición desarticulada es la condición estructural de la conformación de los Estados y las sociedades latinoamericanas y los modos en que estos se relacionan (Zavaleta, 2021). La no totalización orgánica del modo de producción capitalista imposibilita la concentración-separación efectiva y legítima de lo político en el Estado-nación, conformándose Estados aparentes que no han logrado corresponderse exitosamente con una sociedad heterogénea. Esta incapacidad orgánica de dirección significa la persistencia de diversas formas de gobierno y estructuras de autoridad (Tapia, 2010).

La lógica contrainsurgente desplegada por el Estado colombiano busca resolver por medio de la violencia su carácter aparente (Franco, 2009; Estrada, 2015). Para la región del Catatumbo ${ }^{2}$ esto ha significado el uso sistemático de la violencia para la contención de los conflictos sociales, por medio de la acción coordinada o la tolerancia con grupos paramilitares. De ahí que, pese a su riqueza natural, su ubicación estratégica y sus condiciones propicias para la producción agrícola, el Catatumbo se caracterice por el histórico incumplimiento de condiciones sociales mínimas, el pobre apoyo a las actividades económicas, la

2 Se ubica en el extremo nororiental de Colombia y pertenece al departamento de Norte de Santander. Está integrado por los municipios de Ábrego, Convención, El Carmen, El Tarra, Hacarí, La Playa, Ocaña, San Calixto, Sardinata, Teorama y Tibú. Tiene una extensión de 10.089 km2 y hace parte de la frontera terrestre con la República Bolivariana de Venezuela. 
militarización de las relaciones sociales y las pocas garantías para la vida (Minga, 2008; Martínez, 2012; Ascamcat y Ccalcp, 2012; Castellanos, 2017; CNMH, 2018; Estrada et al., 2019). Estas dinámicas, ligadas a la intensificación del conflicto armado, ${ }^{3}$ condujeron a la casi aniquilación de cualquier forma de (re)producción de lo común. Aunque se puede hablar de una memoria larga de la represión en la región, el año 1999 se reconoce como un hito en la vida del Catatumbo, dados sus altos grados de violencia, sus medios atroces para la consecución de sus objetivos y los niveles casi totales de afectación para los catatumberos. ${ }^{4}$

El abigarramiento también tiene que ver con la multisocietalidad o la coexistencia desarticulada de distintos tipos de sociedad (Tapia, 2017). El Catatumbo colombiano ha sido constituido como un territorio intercultural en donde las diferentes formas de vida se relacionan desde sus maneras de organizar y administrar el territorio. ${ }^{5}$ Las trayectorias históricas de (re) producción de lo común en el Catatumbo ${ }^{6}$ ubican rasgos comunes como la forma asamblea para la toma de decisiones, el protagonismo del trabajo colectivo en la economía cotidiana, la importancia de las autoridades comunitarias en la interlocución, dirección y coordinación con otros actores, la coordinación intercomunitaria para la construcción en común de soluciones a los problemas estructurales de la región y la instrumentalización de espacios estatales como la elección de autoridades comunales en alcaldías y concejos con los propósitos de garantizar los avances comunales, fortalecer la perspectiva regional y reclamar al Estado las soluciones a la precariedad generalizada (Ascamcat y Ccalcp, 2012; Castellanos, 2017; CNMH, 2018; Estrada et al., 2019).

En suma, el abigarramiento alude a la "calificación mutua de diversidades económico-sociales de tal suerte que, en concurrencia, ninguna de ellas mantiene su forma (previa)" dada la relación jerárquica o desigual que establecen (Antezana, 2009, p. 132). Estas relaciones no se piensan desde la hibridez, la unidad o la síntesis, lo hacen desde "la coexistencia en paralelo de múltiples diferencias culturales que no se funden, sino que antagonizan y se

3 Si bien el Catatumbo cuenta con una importante presencia de grupos guerrilleros desde la década de 1970, que han establecido diversos grados de relación con las comunidades, algunas signadas por la confrontación, el Estado colombiano ha hecho del Catatumbo un laboratorio para el despliegue de la lucha contrainsurgente de amplio espectro.

4 Durante los años 1999 y 2006, se desplazaron del Catatumbo 99.074 personas, que representan el 59,5\% del total de personas desplazadas de la región en el periodo 1985-2017. Entre las razones que se identifican para la entrada paramilitar al territorio se encuentran eliminar a las guerrillas, controlar el corredor estratégico Córdoba-Bajo Cauca-Sur de Bolivar-Sur del Cesar-Catatumbo, favorecer la explotación de recursos y monocultivos y aniquilar todo proceso de organización social y popular (CNMH, 2018).

5 Actualmente, el Catatumbo está poblado por la comunidad ancestral indígena Barí, con sus resguardos Motlión-Barí y Catalaura-La Gabarra, comunidades campesinas organizadas en el Comité de Integración Social del Catatumbo (CISCA), la Asociación Campesina del Catatumbo (Ascamcat) y el Movimiento por la Constituyente Popular (MCP), así como por múltiples organizaciones locales de transportistas, comerciantes, productores agrícolas y comunidades urbanas, la Unión Sindical Obrera (USO), etc.

6 Estas trayectorias se refieren a los ejercicios de gobierno y trabajo comunitario desarrollados por la comunidad indígena Barí, los sindicatos del petróleo, los partidos y movimientos de izquierda y el llamado movimiento cooperativo campesino desarrollados desde la ancestralidad barí hasta el año 1999. Ver Lozano (2020). 
complementan" las cuales se reproducen y se relacionan de manera contenciosa (Rivera, 2010, p. 71).

El subsuelo político latinoamericano contiene la heterogeneidad social negada pero no subsumida realmente; las distintas formas de vida se relacionan contingentemente entre ellas y con el orden social dominante, desplegando formas de gobierno y autoridad más o menos autónomas, que se fundan en lazos auténticamente comunitarios, con el objetivo de la (re)producción de lo común ${ }^{7}$ para la preservación de la vida humana y no humana (Jiménez y PuelloSocarrás, 2020). Estas sociedades comunitarias se caracterizan por la posesión o propiedad colectiva de la tierra y los medios de producción, correspondientes a una forma de gobierno asamblearia, la poca diferenciación entre los ámbitos sociales y políticos y la poca o nula separación entre comunidad y estructuras de autoridad (Tapia, 2017).

Esta heterogeneidad del subsuelo político abigarrado exige la formulación de categorías analíticas capaces de comprender la multiplicidad de formas en las que se expresan las dinámicas subterráneas de gobierno en sus contextos nacionales, al tiempo que se plantean la explicación de regularidades a escala regional (Tapia y Vega, 2021). En ese sentido, la noción de Habilidades Comunales de Gobierno o Gobernabilidades Comunales (HCG), entendidas como formas de gobierno comunal "desde abajo", que ordenan y administran los territorios y con ellos el trabajo y la vida pretende el análisis de las formas de vida comunitaria en el continente a través de sus prácticas políticas. ${ }^{8}$

Son modalidades autónomas de comando político y administración socioterritoriales que se fundan en cosmovisiones alternas y nativas construidas sobre principios como la desmercantilización, la solidaridad y la reciprocidad (Jiménez y Puello-Socarrás, 2020). De esta manera, las HCG permiten el análisis de mecanismos concretos de gobierno comunitario, proponiendo una aproximación de generalidad en la medida en que articulan dos dimensiones de la (re)producción de lo común: la forma comunitaria de la política y del trabajo en donde la primera dirige y decide sobre la segunda9 ${ }^{9}$ Lozano, 2019; 2020).

7 Lo común entendido como relación social que se produce. Es multiforme y heterogéneo y depende del espacio y tiempo en donde es producido y recreado, así como de las normas que se establecen para su producción (Gutiérrez, 2017). De ahí que se afirme lo "común con comunidad", resaltando el origen comunal de estas relaciones dirigidas para la reproducción de la vida, antes que la "gestión" de "bienes de uso compartido" entre individuos que buscan la maximización de su interés. Esta visión mercantilizadora y privatizadora de lo común, fuertemente impulsada por organismos multilaterales y gobiernos nacionales, justifica y propicia la subsunción de lo comunal al capital (Federici, 2013; Gutiérrez y López, 2019; Jiménez y Puello-Socarrás, 2020).

8 Las HCG asumen la idea de la política como instituyente de lo social, en tanto proceso de decisión sobre la reproducción de la vida (Tapia, 2017; 2020).

9 No obstante, las HCG se encuentran asediadas constantemente por las lógicas del capital. La comunidad se ve en la necesidad de adquirir aquello que no produce, de manera que debe incluirse en la circulación capitalista de mercancías. Se sucede un intercambio desigual de valores producidos según lógicas antagónicas en donde el producto y el trabajo comunitario se convierten en una mercancía más (Bartra, 2006). Aunque el capital explota y refuncionaliza el trabajo comunitario, el análisis desde lo político y la política muestra la capacidad y potencialidad autonómica de lo común en tanto el trabajo comunitario es dirigido, 
La primera tiene que ver con la no enajenación de la soberanía social, en la medida en que la capacidad de decisión sobre lo común radica directamente en la comunidad. Se caracteriza por el recurso a la asamblea como mecanismo para la toma de decisiones, una estructura de autoridad ligada a la idea de servicio regulada por medio de la rotación de cargos. Igualmente, se concreta en ejercicios de soberanía, o expresiones del ser, saber y hacer de la vida comunal (Gutiérrez, 2001 y 2017; Martínez, 2010; Tzul, 2015; Tapia, 2017 y 2019). En el caso del campesinado catatumbero, las Juntas de Acción Comunal (JAC) han funcionado como núcleo del gobierno comunitario, donde se organiza el trabajo comunitario, se resuelven conflictos y se toman decisiones sobre la vida diaria (CNMH, 2018; Estrada et al., 2019).

En segundo lugar, la forma comunitaria del trabajo se desarrolla en medio de una trama comunitaria que lo organiza y dirige. El trabajador no se separa de su producto en la medida en que produce bienes comunitarios basados en el valor de uso y dirigidos a la satisfacción de las necesidades comunales (Gutiérrez y Salazar, 2015). El trabajo comunitario lleva implícita una concepción familiar y comunal de la tierra, cuya apropiación se realiza en función de lo comunalmente decidido (García, 2020). Asimismo, la circulación de bienes comunitarios posibilita la vida material y reproduce el entramado de dispositivos y códigos producidos e interiorizados en los procesos del trabajo. Por último, también tiene que ver con aquellas actividades laborales no remuneradas económicamente centrales en la vida comunitaria como los roles de autoridad, la construcción de espacios comunales, la organización de fiestas, entre otras (Martínez, 2010; Tzul, 2015; Tapia, 2017). En el mundo campesino ${ }^{10}$ colombiano, estas formas se han desarrollado en procesos de colonización y ampliación de la frontera agrícola bajo actividades como el trabajo a "vuelta de mano" o "manos prestadas”, los convites o las juntas campesinas (CNMH, 2018).

\section{Metodología}

La selección del caso tuvo en cuenta la prevalencia en los estudios latinoamericanos del análisis de formas comunitarias de vida indígenas especialmente en contextos andinos y el vacío en el ámbito nacional del abordaje de experiencias campesinas desde la reproducción de lo común. ${ }^{11}$ Se propuso

orientado y comandado de diversas formas que escapan el control del capital (Gutiérrez y Salazar, 2015). Así la reproducción comunitaria implique ceder mayor o menor cantidad de bienes producidos, dicha decisión es política. Como lo demuestra la problemática de los cultivos de uso ilícito en la comunidad campesina del Catatumbo, la comunidad genera propuestas encaminadas a subvertir las situaciones que la obligan a entrar en este mercado capitalista ilegal (Ascamcat y Ccalcp, 2012).

10 El campesinado es asumido en tanto clase social constituida y constituyente, consustancialmente diversa fundada en un proyecto común de resistencia, basada en una economía familiar comunitaria (Bartra, 2011)

11 En el ámbito nacional, se privilegian los análisis centrados en el concepto de territorio, desde estudios relacionados con una visión hegemónica del territorio como agregación de individuos, hasta aquellas aproximaciones críticas que observan en los ejercicios de organización territorial campesina como las zonas de reserva campesina la expresión de una territorialidad productora y contenedora de relaciones 
una mirada analítica sobre los momentos históricos donde el antagonismo social se hace más evidente ya que permiten no solo dar cuenta de los deberesser planteados intrínsecamente por los sujetos que actúan, sino también del impacto que dichas acciones tienen en la totalidad social (Gutiérrez, 2008). Así, la temporalidad que se ubicó en la investigación fue el período comprendido entre los años 2005, año de constitución de la Asociación Campesina del Catatumbo-Ascamcat, y 2018, fecha de la última Audiencia Regional Popular, máximo escenario de decisión comunitaria campesina.

La investigación se planteó según los principios de la investigación social comprometida y el diálogo de saberes, asumiendo la comunidad campesina del Catatumbo como productora de conocimientos y como protagonista de su propia historia (Fals Borda, 2015). Se tomaron en cuenta cinco fuentes de documentación: materiales producidos por Ascamcat y la comunidad campesina (comunicados, actas, circulares, propuestas y memorias); revisión de prensa relativa a los procesos señalados; actas de la Mesa de Interlocución y Acuerdo del Catatumbo (MIA-C); ${ }^{12}$ entrevistas semiestructuradas realizadas a autoridades de Ascamcat; y observación participante, durante la reunión de Equipos de Apoyo de Ascamcat en junio de 2017 y la Tercera Audiencia Popular Regional de Ascamcat en agosto de 2018.

Igualmente, se dio cuenta de tres niveles o momentos de significación política interrelacionados (Tapia, 1992). Un primer nivel referido a la "conciencia de la producción o articulación de la subjetividad [...], [de] colocarse en el mundo, con los otros, con iniciativas y opiniones" (Tapia, 1992, p. 18) y que alude a las acciones de decisión y dirección colectiva que ocurren en lo intracomunal. Un segundo nivel extracomunal comprende la articulación de varias prácticas y sujetos en la totalidad social, en la cual las diferentes acciones políticas se encuentran, con diferentes niveles de modificación, transformación o influencia mutua. El tercer nivel se refiere a la vuelta de la acción y su sentido luego de su encuentro con otras acciones y sentidos. Este nivel tiene que ver con las transformaciones o modificaciones que sufren las prácticas en su encuentro en lo extracomunal y que remiten nuevamente a la autonomía relativa de lo intracomunal.

La apuesta metodológica comprendió la acción política y su sentido campesinos que se despliegan según objetivos, prácticas organizativas y en general según una concepción de mundo particular y en un espacio delimitado, que implica comprensiones sobre quién tiene derecho a hacer política o quién debe hacerlo, los límites, forma, contenido y mecanismos de la misma (Tapia, 1992). Así, la forma de la política en la comunidad campesina del Catatumbo se

sociales alternativas. Si bien esto permite ver las formas de administración, apropiación y organización del espacio, se subestiman los procesos que dan origen a dichas prácticas. A través del rastreo de lo común y su (re)producción, se alude a las formas políticas de definición de la vida a futuro y sus disposiciones, entre las que se cuenta el ordenamiento del espacio apropiado, pero no solamente.

12 Especialmente los relativos a los Acuerdos de Confianza y la Estrategia para la Post Erradicación y Fase Inicial de la Intervención para la Estabilización Socio Económica como parte del Proceso de Sustitución y Desarrollo Alternativo. 
analizó metodológicamente por medio de tres indicadores: el proceso de toma de decisiones, la estructura de autoridad y los ejercicios de soberanía. Por su parte, el trabajo comunitario se leyó desde la relación que se establece con la tierra, la producción y circulación de los bienes comunitarios y las formas concretas de trabajo no remunerado, teniendo en cuenta la articulación de ambos niveles, en donde la primera dirige y organiza el segundo. ${ }^{13}$

\section{Las Habilidades Comunales de Gobierno campesino en el Catatumbo, 2005-2018}

Aunque para principios del siglo XXI se asegura la desestructuración del tejido social catatumbero (Castellanos, 2017; CNMH, 2018), las comunidades logran confluir nuevamente en escenarios de organización de la vida comunal. En el año 2005 se funda la Asociación Campesina del Catatumbo-Ascamcat a raíz de nuevos desplazamientos causados por las dinámicas del conflicto armado. Ascamcat surge como reencuentro de la comunidad campesina para la reconstrucción de sus relaciones comunitarias, la defensa y permanencia en el territorio, la sustitución de cultivos de uso ilícito y la solución a los factores socioeconómicos ligados a su origen, la participación en la toma de decisiones sobre el campo y el respeto a la vida y los Derechos Humanos (DDHH) de los y las catatumberas.

De manera que Ascamcat ${ }^{14}$ no solamente es una organización para la defensa y promoción de los intereses campesinos, sino que es la condensación de la forma política comunitaria, en tanto dirige los procesos de trabajo comunitario o de reproducción material de la vida en un contexto de conflicto armado, actúa en la interlocución y negociación frente al Estado colombiano y otros actores del territorio y organiza la acción política campesina. El gobierno de Ascamcat está íntimamente ligado a la consecución y materialización de la Zona de Reserva Campesina del Catatumbo ${ }^{15}$ (ZRC-C) como expresión de la territorialidad campesina ${ }^{16}$ y que, frente a la falta de reglamentación por parte del Estado,

13 La información fue analizada a partir del rastreo de referenciales entendidos como aquellos "conjunto[s] de representaciones que se tienen sobre la realidad y desde la cuales se busca explicarla, intervenirla y coproducirla" por parte de las sociedades (Lozano, s. f., p. 1).

14 Ascamcat organiza el gobierno campesino bajo tres criterios transversales: el carácter biofísico de la región que divide el territorio en Alto, Medio y Bajo Catatumbo; la división de tareas y responsabilidades según carteras, semejantes a los ministerios del gobierno convencional; y la asamblea como mecanismo de toma de decisiones. Su estructura tiene en su base los llamados comités veredales, que a su vez conforman niveles intermedios de comunicación y de autoridad, supeditados a la decisión de las asambleas locales y a lo acordado en la Audiencia Regional Popular.

15 Las Zonas de Reserva Campesina son una figura de ordenamiento territorial especial reconocidas y reglamentadas por el Estado colombiano para el fomento de la economía campesina, regular la ocupación de baldíos y garantizar las condiciones sociales de vida campesina (Ley 160 de 1994 y Decreto 1777 de 1996). Son producto de las luchas emprendidas a mediados de los años noventa del siglo XX y han sido apropiadas en las banderas, agendas y reivindicaciones del campesinado colombiano (Estrada, 2013).

16 Relacionada con sentidos de complementariedad con la naturaleza, la protección de todas las formas de vida, el autosostenimiento de la economía campesina y las plenas condiciones para las comunidades y organizaciones de decidir activamente sobre el futuro del Catatumbo (Estrada et al., 2019). 
funciona hoy de manera autónoma y de facto. En ese sentido, durante el período 2005-2018, Ascamcat desplegó cinco HCG: la asamblea, la Guardia Campesina, el Refugio Humanitario, la campaña y la Mesa de Interlocución y Acuerdo (MIA-C). ${ }^{17}$

La asamblea campesina surge como espacio de dirección de lo común y funciona en los niveles veredales, locales y regionales. La Audiencia Regional Popular es el máximo espacio de decisión comunal, donde se elige la Junta Directiva, que tiene un papel de coordinación de la acción política y de vocería en espacios de negociación y se definen los derroteros de la comunidad para un periodo de 4 años. Además, las asambleas se ocupan del desarrollo de proyectos productivos, campañas de educación, administración de bienes comunitarios, la construcción de espacios comunales, la provisión de servicios básicos, el arreglo de vías interveredales y brigadas de salud, entre otros (Theseus Unal, 2018). En ocasiones incluyen la participación de representantes del Estado para la interlocución y la elaboración de acuerdos. Por medio del gobierno asambleario, la comunidad campesina se ha encontrado con otras formas de vida en procesos de sustitución de cultivos de uso ilícito y la construcción de un territorio intercultural con reconocimiento de propiedades familiares, comunitarias e interculturales de la tierra.

En segundo lugar, la Guardia Campesina del Catatumbo está constituida como mecanismo de autoprotección y su acción se fundamenta en el diálogo, la interlocución y la defensa del territorio. Tiene un carácter voluntario, civil y de promoción y defensa de los DDHH y está conformada por "personas de la más alta estima y del mayor reconocimiento comunitario" (PoderPaz et al., 2015, p. 14). Su autoridad radica en la dignidad campesina representada en el Bolillo Patriótico. ${ }^{18}$ La Guardia está organizada para ejercer la soberanía campesina en el territorio y sirve como espacio de formación de autoridades comunales y de cualificación comunitaria a través de escuelas. Además, se encarga de cuidar y proteger los distintos espacios de la vida comunitaria como asambleas, marchas, tomas, bloqueos, así como momentos de esparcimiento y asume la interlocución con actores armados estatales y no estatales. Estos ejercicios de autoridad están fuertemente ligados a un sentimiento de reconocimiento y confianza comunales y de responsabilidad con la comunidad en tanto su actuación se supedita a los lineamientos acordados en las asambleas.

En tercer lugar, los refugios humanitarios tienen un carácter extraordinario y se desarrollan en momentos de amenaza a la vida comunal, generalmente relacionados con la agudización del conflicto armado. Su objetivo es la preservación de la comunidad y su permanencia en el territorio, por medio

17 Para una ampliación, ver Lozano (2019 y 2020) y Chica et al. (2021).

18 Cada guardia campesino porta un Bolillo Patriótico, decorado con los colores amarillo, azul y rojo, un pequeño machete que simboliza el trabajo campesino y la inscripción "Vuelve Bolívar". Por último, posee cuatro nudos de hilo que simbolizan los cuatro campesinos asesinados durante el Paro del Catatumbo de 2013 (Estrada et al., 2019). 
de la intensificación de los procesos de gobierno y trabajo comunitarios. Los refugios se organizan en un lugar determinado donde las familias campesinas conviven hasta que se decida su levantamiento. Cuentan con un economato, cocina y comedores comunales, enfermería, espacios de encuentro, formación política y recreación. Por medio de los refugios, se ha evitado la profundización del desarraigo gracias a la reafirmación de los lazos comunitarios en el día a día y son espacios para el encuentro con instituciones y otros actores.

Por su parte, las campañas tienen por objetivos la verificación y el seguimiento a las violaciones de DDHH, se realizan por iniciativa comunal para acompañar los territorios afectados y con la participación de organizaciones civiles y comunidades amigas. Además de visibilizar las condiciones de violencia de la región, las campañas realizan veeduría y control sobre las acciones de la fuerza pública y articulan propuestas de salida a las situaciones de violencia. También existen campañas de educación y formación de autoridades comunales y talleres de sensibilización frente a diversos temas como la historia de la movilización social en la región, herramientas de exigibilidad de derechos y la defensa de la ZRC-C y el territorio.

Por último, la Mesa de Interlocución y Acuerdo MIA-C se conformó como mecanismo de encuentro y diálogo entre el Estado y la comunidad. En ella participan los voceros campesinos ${ }^{19}$ junto con voceros de otras comunidades y representantes del gobierno nacional, con el propósito de generar proyectos y soluciones sobre cuatro temas específicos: crisis Humanitaria y DDHH, tierra y territorio, recursos naturales y cultivos de uso ilícito, que se tratan en cuatro momentos. Un primero de preparación intracomunitaria de los temas entre voceros y técnicos especializados acompañantes de Ascamcat, donde se construyen propuestas en forma de borradores. El momento de encuentro técnico se refiere al encuentro entre el equipo técnico de Ascamcat y representantes del gobierno para construir borradores conjuntos. El momento político o de encuentro para la negociación construye los acuerdos por consenso y está acompañado por garantes internacionales. Las partes cuentan con un espacio autónomo de consulta y se tiene por acuerdo detener la discusión cuando esta alcanza puntos álgidos para no afectar el ambiente de la MIAC. Finalmente, el momento de socialización se refiere a la responsabilidad de los voceros de informar a las comunidades sobre lo discutido y lo acordado, para recoger propuestas o disensos, a través de emisoras comunitarias, redes sociales, talleres y los órganos informativos de Ascamcat como "Relámpago TV" y el boletín "Relámpago".

Aunque el proceso de la MIA-C ha sido accidentado por la negativa de los gobiernos a asistir al espacio, ha logrado la puesta en marcha de proyectos que reconocen la autoridad campesina para la definición de la población y veredas objeto de los beneficios sociales, la contratación de mano de obra local y la

19 Los voceros de Ascamcat tienen como guía las propuestas contenidas en el Plan de Desarrollo Sostenible de la ZRC-C. Ver Ascamcat y Ccalcp (2012). 
veeduría constante por parte de los comités veredales en el cumplimiento de las obras.

En suma, el caso de Ascamcat como forma política de la comunidad campesina del Catatumbo denota el despliegue de HCG para la dirección del trabajo comunitario para la (re)producción de lo común en condiciones particulares. De esta manera, la protección de la vida campesina implica asumir el principio político del diálogo para la resolución de conflictos inter e intra-comunitarios, la exigencia frente al Estado en la consecución de garantías mínimas y la consecución de nuevos espacios que amplíen la democracia e incluyan la diversidad negada.

\section{Conclusiones}

El análisis del subsuelo político exige la reinvención o actualización del pensamiento crítico que pretende explicaciones sobre sus dinámicas. Esto implica asumir en primera medida su carácter profundamente diverso, sus múltiples formas de manifestación, crítica y contención del orden social hegemónico. En segunda medida, en condiciones de abigarramiento, es necesaria una aproximación al subsuelo político con categorías intermedias que tengan en cuenta las especificidades de las relaciones entre la multisocietalidad y Estados aparentes incapaces de corresponder a dicha heterogeneidad social. Este carácter intermedio está dado por su capacidad de tener en cuenta las especificidades de las formaciones sociales locales a la vez que se piensan las regularidades continentales en un sentido de síntesis.

De ahí que la categoría de Habilidades Comunales de Gobierno avance en ese propósito, planteando la articulación de las dimensiones políticas y del trabajo, en donde la primera dirige la segunda como regularidad de las formas de vida comunitaria en América Latina. Al mismo tiempo, permite la explicación de esta articulación y sus formas de despliegue en la especificidad de contextos nacionales. En el caso del Catatumbo, se observan unas dinámicas históricas de reproducción de lo común, con altos grados de autonomía, que debieron enfrentar la acción aniquiladora del Estado colombiano, el cual no ha sido capaz de subsumir totalmente las formas de vida que perviven en el territorio.

En ese sentido, frente a los retos de pensar la comunicación en el ámbito latinoamericano (Herrera, Sierra y del Valle, 2016), las HCG permiten acercarse a las prácticas comunitarias de comunicación que se despliegan y que son propias del subsuelo político. Los ejercicios de comunicación de Ascamcat tienen que ver, en primer lugar, con la rendición de cuentas por parte de las autoridades campesinas frente a su comunidad, en la medida en que a través de ellos se presentan los avances y resultados de los espacios de negociación con el Estado colombiano, así como el estado de los programas comunitarios dirigidos por la Asociación referidos a la adecuación de vías, la construcción de espacios comunales o de proyectos de ganadería o de producción agrícola, entre otros. 
En segundo lugar, los medios comunitarios de Ascamcat contribuyen a mantener y fortalecer la relación entre autoridades y comunidades, en ejercicios de transparencia, de modo que se evalúa constantemente las labores de vocerías y dirección, según las necesidades comunitarias. Por último, la comunidad campesina comunica también para el nivel extra comunitario puesto que es de vital importancia la denuncia constante de las situaciones derivadas del conflicto social y armado en la región, relacionadas con las infracciones a los DDH y al DIH por parte de los actores armados, así como la visibilización de las iniciativas comunitarias desarrolladas, contrarrestando la narrativa oficial y hegemónica del Catatumbo como una "zona roja” o de guerra.

En suma, la comunicación también tiene un sustrato comunitario vital para el gobierno campesino y se despliega con una finalidad de preservación, protección y defensa del territorio y la vida comunal campesina, bajo sentidos alternativos a la comunicación para la dominación o la mercantilización. Por tanto, es necesario insistir en el diálogo de saberes entre las diferentes formas de vida en contextos de abigarramiento, en un sentido de la comunicación como proceso intersubjetivo de construcción de sentido (Acosta y Garcés, 2016). Esto significa comprender las matrices societales sobre las cuales se originan las prácticas de comunicación y el respeto a sus autonomías, el reconocimiento en la esfera nacional de la pluralidad, la diversidad y la heterogeneidad de las sociedades latinoamericanas y la necesidad de transformar las situaciones estructurales que marginan en el subsuelo a formas de vida potencialmente o de facto contrahegemónicas.

En conclusión, desde el despliegue del gobierno campesino, la comunidad del Catatumbo ha logrado rehacer los vínculos comunitarios destruidos por la acción estatal y para-estatal, construyendo alternativas de vida al capital. El carácter contingente del abigarramiento alude precisamente a esta condición; la incapacidad del orden social de hacerse totalmente orgánico remite a las resistencias tejidas desde la ancestralidad que se actualizan y confrontan las lógicas de la valorización del valor y que hoy desatan los movimientos telúricos del subsuelo político, trazando horizontes políticos de vida y emancipación.

\section{Referencias bibliográficas}

Acosta, G. y Garcés, A. (2016). El diálogo de saberes en comunicación: reconfiguraciones de la formación y de la investigación. Anagramas Rumbos y sentidos de la comunicación. 15(29). Medellín: Universidad de Medellín. 17-23.

Antezana, L. (2009). Dos conceptos en la obra de René Zavaleta Mercado: Formación abigarrada y democracia como autodeterminación. En Olivé, L. et al. Pluralismo epistemológico (pp. 117-142). La Paz: CLACSO / Muela del Diablo Editores / Comunas / CIDES / UMSA.

Ascamcat y Ccalcp (Asociación Campesina del Catatumbo y Corporación Colectivo de Abogados Luis Carlos Pérez (Ccalcp). (2012). Plan de desarrollo sostenible para la constitución de la Zona de Reserva Campesina del Catatumbo. Bucaramanga. 
Bartra, A. (2006). El capital en su laberinto, De la renta de la tierra a la renta de la vida. Ciudad de México: UACM / ITACA / CEDRSSA.

Bartra, A. (2011). Tiempo de mitos y carnaval. Indios, campesinos y revoluciones. De Felipe Carrillo Puerto a Evo Morales. Ciudad de México: ITACA / PRD DF.

Castellanos, C. (2017). Nociones de despojo y restitución de la autonomía del campesinado catatumbero. Relatos de la Asociación Campesina del Catatumbo (Ascamcat). Tesis de maestría, Universidad Industrial de Santander. Recuperado de https://bit.ly/2Dzueb9

Chica, L.; Chicaíza, J. y Lozano, A. (2021). La territorialización de paz y la reproducción de lo común en el Catatumbo colombiano. (Artículo inédito). Buenos Aires: CLACSO / Universidad Nacional de Colombia.

Centro Nacional de Memoria Histórica-Colombia (CNMH). (2018). Catatumbo: memorias de vida y dignidad. Bogotá: CNMH.

Estrada, J. (comp.). (2013). Territorios campesinos: la experiencia de las zonas de reserva campesina. Bogotá: Universidad Nacional de Colombia (sede Bogotá) / Instituto Colombiano de Desarrollo Rural Incoder.

Estrada, J. (2015). Acumulación de clase, dominación de clase y rebelión armada. Elementos para una interpretación histórica del conflicto social y armado. Informe presentado a la Comisión Histórica del Conflicto y sus Víctimas. Bogotá: Espacio Crítico.

Estrada, J.; Jiménez, C. y Puello-Socarrás, J. (comp.) (2019). Catatumbo resiste. Cincuenta y tres días de Paro. Bogotá: Universidad Nacional de Colombia.

Estrada, J.; Jiménez, C. y Puello-Socarrás, J. (2020). Contra Nuestra América. Estrategias de la derecha en el siglo XXI. Buenos Aires: CLACSO.

Fals Borda, O. (2015). Una sociología sentipensante para América Latina. Buenos Aires: CLACSO / Siglo XXI.

Federici, S. (2013). Revolución en punto cero. Trabajo doméstico, reproducción y luchas feministas. Traducción Scriptorium. Madrid: Traficantes de Sueños.

Franco, V. (2009). Orden contrainsurgente y dominación. Bogotá: Siglo del Hombre / Instituto Popular de Capacitación.

García, A. (2020). Forma valor y forma comunidad: Aproximación teórico-abstracta a los fundamentos civilizatorios que preceden al Ayllu Universal. Buenos Aires: CLACSO / Prometeo.

Gutiérrez, R. y Salazar, H. (2015). Reproducción comunitaria de la vida. Pensando la trans-formación social en el presente. El Apantle. Revista de Estudios Comunitarios. 1. Puebla: Sociedad Comunitaria de Estudios Estratégicos, 15-50.

Gutiérrez, R. y López, C. (2019). Producir lo común para sostener la vida. Notas para entender el despliegue de un horizonte comunitario-popular que impugna, subvierte y desborda el capitalismo depredador. En Gabbert, K. y Lang, M. ¿̇Cómo se sostiene la vida en América Latina? Feminismos y re-existencias en tiempos de oscuridad (pp. 387-417). Quito: Fundación Rosa Luxemburg / Abya-Yala.

Gutiérrez, R. (2001). Forma comunal y forma liberal de la política: de la soberanía social a la irresponsabilidad civil. En. García, A., Gutiérrez, R., Prada, R., Tapia, L. Pluriverso. Teoría política boliviana (pp. 57-73). La Paz: Colección Comuna / Muela del Diablo Editores.

Gutiérrez, R. (2008). Los ritmos del Pachakuti. Movilización y levantamiento popular-indígena en Bolivia (2000-2005). Buenos Aires: Tinta Limón.

Gutiérrez, R. (2017). Horizontes comunitario-populares. Producción de lo común más allá de las políticas estado-céntricas. Madrid: Traficantes de Sueños. 
Herrera, E.; Sierra, F. y Del Valle, C. (2016). Hacia una Epistemología del Sur. Decolonialidad del saber-poder informativo y nueva Comunicología Latinoamericana. Una lectura crítica de la mediación desde las culturas indígenas. Chasqui. Revista Latinoamericana de Comunicación 131.Quito, CIESPAL, 77-105.

Jiménez, C. y Puello-Socarrás, J. F. (2020). Two faces of Commons. Communal forms of government from below as counter-hegemonic alternatives. Ephemera. Theory \& politics in organization. 20(1), Feb. ISSN 1473-2866, 17-50.

Lozano, A. (s. f.). Referenciales de política pública (orientaciones generales, trabajo de campo). Documento de trabajo.

Lozano, A. (2019). Gobernabilidad y (re)producción de formas de vida comunitaria en el Catatumbo colombiano. Cuadernos Americanos 168. México, 2019 / 2, 57-85.

Lozano, A. (2020). El gobierno en condiciones de abigarramiento. Las Habilidades Comunales de Gobierno de la Asociación Campesina del Catatumbo, 2005-2018. Tesis de Maestría, Universidad Nacional de Colombia. Recuperado de https://repositorio.unal. edu.co / handle / unal / 79408

Martínez, P. (2012). Tendencias de acumulación, violencia y desposesión en la región del Catatumbo. Revista de Ciencia Política. 12, 113-149.

Martínez J. (2010). Eso que llaman comunalidad. Oaxaca: Culturas Populares, Conacultura / Secretaría de Cultura, Gobierno de Oaxaca / Fundación Alfredo Harp Helú.

Minga y Fundación Progresar. (2008). Memoria: puerta a la esperanza. Violencia sociopolítica en Tibú y El Tarra. Región del Catatumbo 1998-2005. Bogotá: Minga / Fundación Progresar.

Ouviña, H. (2020). El Estado y la reactivación del Ciclo de Impugnación al Neoliberalismo en América Latina (2019-2020). En Varios. Estados alterados. reconfiguraciones estatales, luchas políticas y crisis orgánica en tiempos de pandemia (pp. 259-284). Buenos Aires: CLACSO / Muchos Mundos Ediciones / IEALC.

Poder Paz (Corporación Construyendo Poder, Democracia y Paz), Ccalcp (Corporación Colectivo de Abogados Luis Carlos Pérez), Constituyentes por la Paz con Justicia Social. (2015). Constituyente Regional de Catatumbo. Municipio del Tarra. Norte de Santander. Colombia. 5 y 6 de diciembre de 2014 .

Rivera, S. (2010). Ch’ixinakax utxiwa: una reflexión sobre prácticas y discursos descolonizadores. Buenos Aires: Tinta Limón.

Tapia, L. (1992). Politización. Ensayos teórico-metodológicos para el análisis político. La Paz: CIDES / UMSA.

Tapia, L. (2008). Política Salvaje. La Paz: CIDES / UMSA / Muela del Diablo Editores / CLACSO.

Tapia, L. (2010). El Estado en condiciones de abigarramiento. En García A., Prada, R., Tapia, L. y Vega, O., (2010). El Estado. Campo de Lucha. La Paz: CLACSO / Muela del Diablo / COMUNA.

Tapia, L. (2013). De la forma primordial a América Latina como horizonte epistemológico. La Paz: CIDES / UMSA / Autodeterminación.

Tapia, L. (2017). Marxismo Transcrítico. La Paz: Autodeterminación.

Tapia, L. (2019). Principios organizativos de la política: estado y comunidad. En Makarán, G. López, P. y Wahren, J. (coord.). Vuelta a la autonomía. Debates y experiencias para la emancipación social desde América Latina (pp. 81-10o). Ciudad de México: Bajo Tierra A. C. / Centro de Investigaciones sobre América Latina y el Caribe / Universidad Nacional Autónoma de México. 
Tapia, L. y Vega, O. (2021). Presentación de René Zavaleta Mercado. En Zavaleta, R. Horizontes de visibilidad. Aportes latinoamericanos marxistas. Obras escogidas (pp. 11-30). Madrid: Sylone / Traficantes de sueños.

Theseus Unal. (18 de noviembre de 2018). Guardia Campesina en el Catatumbo [video]. YouTube. https://bit.ly/33OsNAm

Tzul, G. (2015). Sistemas de gobierno comunal indígena: la organización de la reproducción de la vida. El Apantle I. Revista de Estudios Comunitarios. Puebla: SOCEE, 145-19o.

Zavaleta, R. (2021). Horizontes de visibilidad. Aportes latinoamericanos marxistas. Obras escogidas. Madrid: Sylone / Traficantes de sueños. 